\title{
Influence of substrate temperature on the structural and optical properties of crystalline ZnO films obtained by pulsed spray pyrolysis
}

\author{
Oleksandr Dobrozhan, ${ }^{a *}$ Denys Kurbatov, ${ }^{a}$ Anatoliy Opanasyuk, \\ Hyeonsik Cheong ${ }^{b}$ and Andreu Cabot ${ }^{c}$
}

In this work, we report on the structural and optical properties of ZnO films deposited by pulsed spray pyrolysis at relatively low temperatures, compatible with a large variety of substrates and processing technologies. Crystalline ZnO films were deposited onto glass substrates using zinc acetate dihydrate dissolved in distilled water with concentration of $0.2 \mathrm{M}$. The temperature of the substrate was varied in the range $T_{s}=473-673 \mathrm{~K}$ with $\Delta T=50 \mathrm{~K}$. Effect of $T_{s}$ were investigated by scanning electron microscopy, $\mathrm{x}$-ray diffraction and energy dispersive $x$-ray, and optical spectroscopies. Also, the influence of $T_{s}$ on the grain size, phase composition, texture quality, coherent scattering domain size, crystal lattice parameters, chemical composition, transmission coefficient, and the bang gap of the ZnO films were studied. X-ray diffraction analysis revealed that films were polycrystalline with hexagonal phase and showed as preferential orientation (101) at $T_{s}<573 \mathrm{~K}$ and (100) and (002) at $T_{s}>573 \mathrm{~K}$. Scanning electron microscopy (SEM) measurements showed that the substrate temperature has a strong effect on morphology of the films. Energy dispersive analysis revealed that $\mathrm{ZnO}$ films consisted of the non-stoichiometric compounds. Optical measurements showed ZnO films to be highly transparent in the visible region, and optical band gap is shifting from $3.18 \mathrm{eV}$ to $3.30 \mathrm{eV}$. Copyright $\odot 2015$ John Wiley \& Sons, Ltd.

Keywords: thin films; pulsed spray pyrolysis; ZnO; structural properties; optical properties

\section{Introduction}

Zinc oxide $(\mathrm{ZnO})$ is a direct band gap n-type semiconductor $\left(E_{g}=3.37 \mathrm{eV}\right.$ at $T=300 \mathrm{~K}$ ) with the highest exciton binding energy $(60 \mathrm{eV})$ among binary compounds. ${ }^{[1,2]}$ Due to its unique physical, electrical and optical properties, and its nontoxicity, chemical and thermal stability in ambient atmosphere, $\mathrm{ZnO}$ is a promising candidate for use in micro-electronics and nanoelectronics, ${ }^{[3]}$ optoelectronics, ${ }^{[4]}$ sensors $^{[5,6]}$, and photovoltaic devices, ${ }^{[7,8]}$ among others. As $\mathrm{ZnO}$ does not contain rare elements in its structure and can be obtained with inexpensive methods, when strongly doped, it is also an alternative to traditional transparent conductive materials such as ITO $\left(\left(\mathrm{In}_{2} \mathrm{O}_{3}\right)_{0.9}-\left(\mathrm{SnO}_{2}\right)_{0.1}\right)$ and $\left.\mathrm{FTO}\left(\mathrm{SnO}_{2}: \mathrm{F}\right)\right)$ for use in thin films solar cells. ${ }^{[9]}$ Besides, $\mathrm{ZnO}$ can also be used as an antireflection or a window layer in solar cells. ${ }^{[10]}$

A wide range of methods including magnetron sputtering, ${ }^{[11]}$ chemical vapor deposition, ${ }^{[12]}$ thermal evaporation, ${ }^{[13]}$ chemical bath deposition, ${ }^{[14]}$ spray pyrolysis, ${ }^{[15,16]}$ hydrothermal growth, ${ }^{[17,18]}$ and electrodeposition ${ }^{[19-23]}$ is used to prepare $\mathrm{ZnO}$ thin films. Among them, spray pyrolysis is a particularly simple, versatile, low-cost, and highly scalable method to deposit solid, porous, and nanostructured films on potentially large area substrates made of different materials. The properties of the thin films prepared by this method depend on the choice of precursors and on the physical and technical conditions of their deposition. One particularly important parameter, both for its influence on the film properties but also because of the limitations it imposes on the substrate material, is the deposition temperature. ${ }^{[24,25]}$ The substrate temperature needs to be minimized to prevent alloying and compositional grading between the $\mathrm{ZnO}$ and material at the surface of the substrate. Low deposition temperatures are also necessary to make the process compatible with polymeric substrates. A third obvious advantage of a low deposition temperature is a reduction of the processing costs. However, low substrate temperatures normally result in poor quality films.

Very recently, M. Ortel et al. have reported the use of a pulsed spray pyrolysis technique to prepare high performance $\mathrm{ZnO}$ thin films. ${ }^{[26]}$ They experimentally demonstrated that films with similar performance can be obtained at a significantly lower deposition temperature by means of a pulsed deposition. This reduction of the growth temperature at equal performing layers was associated with a more effective outward transport of by-products and inward diffusion of oxygen in the pulsed mode. While in their excellent paper, M. Ortel et al. detailed the influence of the substrate temperature on the charge transport properties of the films; a description of the structural properties of the $\mathrm{ZnO}$ films deposited by pulsed spray deposition as a function of the substrate temperature is still missing.

* Correspondence to: O. Dobrozhan, Department of Electronics and Computer Technology, Faculty of Electronics and Informational Technologies, Sumy State University, 2, Rymskogo-Korsakova st., Sumy, 40007, Ukraine.

E-mail:dobrozhan.a@gmail.com

a Department of Electronics and Computer Technology, Faculty of Electronics and Informational Technologies, Sumy State University, 2, Rymskogo-Korsakova st., Sumy, 40007, Ukraine

b Department of Physics, Sogang University, Seoul, 121-742, Korea

c Institució Catalana de Recerca i Estudis Avançats - ICREA, Barcelona, 08930, Spain 
In this paper, we investigate the influence of the substrate temperature on the structural, substructural, and optical properties of zinc oxide films deposited by the pulsed spray pyrolysis method, focusing in the low temperature range, $475 \mathrm{~K}<T_{s}<675 \mathrm{~K}$.

\section{Experimental details}

A custom-made laboratory pulsed spray deposition setup, which schematic diagram is shown in Fig. 1, was used to obtain $\mathrm{ZnO}$ films. ${ }^{[27]}$ Briefly, it consists of a heated support for the sample substrate, a thermocouple for registering the values of the substrate temperature, a spray gun, comprising a reservoir for the initial solution, an on/off valve and a sprayer nozzle to atomize the solution, and a compressor that provides the air flow at sufficient pressure to transport dispersed precursor particles to the heated substrate.

To produce $\mathrm{ZnO}$ films, a solution of zinc acetate dihydrate $\mathrm{Zn}\left(\mathrm{CH}_{3} \mathrm{COO}\right)_{2} \cdot 2 \mathrm{H}_{2} \mathrm{O}$ and distilled water with a concentration of $0.2 \mathrm{M}$ was used as a precursor. In order to increase the $\mathrm{Zn}\left(\mathrm{CH}_{3} \mathrm{COO}\right)_{2} \cdot 2 \mathrm{H}_{2} \mathrm{O}$ solubility, a few drops of $\mathrm{HCl}$ were added to the initial solution. The obtained solution was sprayed onto glass substrates with $25 \times 25 \times 1 \mathrm{~mm}$ size, which had been cleaned beforehand with ethanol. The temperature of the substrate was varied in the range $T_{s}=473-673 \mathrm{~K}$ with $\Delta T=50 \mathrm{~K}$. The distance between the spray nozzle and substrate surface was set to $20 \mathrm{~cm}$. Airflow with pressure of $0.2 \mathrm{MPa}$ was used as the carrier of the dispersed precursor particles. The rate of film deposition was $2 \mathrm{ml} / \mathrm{min}$, and the volume of sprayed solution was set to $5 \mathrm{ml}$ per sample. For the formation of films without oversaturation of precursor near the heated substrate surface and to facilitate by-product and oxygen transport, a pulsed spraying mode was used. Spraying pulse duration was set to $2 \mathrm{~s}$, and the time between pulses was set to $3 \mathrm{~s}$. Thirty pulses were used to deposit each layer.

The surface morphology of the $\mathrm{ZnO}$ films was characterized by means of scanning electron microscopy with a Zeiss Auriga microscope (Zeiss, Oberkochen, Germany). The films thicknesses were measured from the cross-sectional SEM images. The chemical composition of the films was determined by energy dispersive analysis (EDX) using $x$-rays inside SEM, for which a $20-\mathrm{kV}$ accelerating voltage and a $1.5-\mu \mathrm{m}$ penetration depth of electrons into the films were used. Additional peaks from substrate elements were deducted from the EDX spectra. The atomic concentrations of the elements $\left(C_{Z n}, C_{O}\right)$ were measured at least at three different points of the sample surface, and the average value was used. Stoichiometry of the ZnO films was calculated using the correlation $\gamma=\frac{C_{0}}{C_{z n}}$.

$\mathrm{X}$-ray diffraction analysis was used to study the structural properties of the ZnO layers. ${ }^{[28-32]}$ A Bruker D8 x-ray diffractometer (Bruker, Optics Inc., Billerica, MA, USA) with the $\mathrm{K}_{\alpha}$ radiation of a

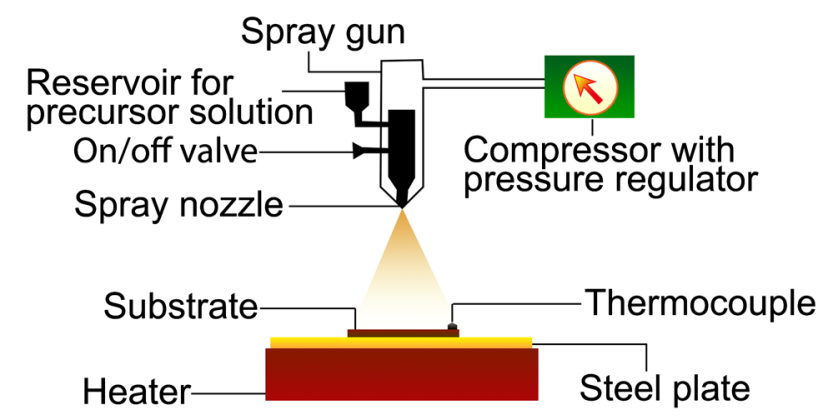

Figure 1. Schematic diagram of the custom-made laboratory setup for obtaining $\mathrm{ZnO}$ thin films by pulsed spray pyrolysis.
Cu anode $(\lambda=0.15406 \mathrm{~nm}, U=40 \mathrm{kV}$, and $I=40 \mathrm{~mA})$ was used. The diffraction patterns were normalized by the intensity of the hexagonal phase (101) peak. The phase analysis was carried out by comparing relative intensities of the samples with those of an etalon. ${ }^{[28]}$

The quality of the film texture was estimated by the Harris method that is convenient in the investigation of planar samples with the texture axis oriented normal to its surface. ${ }^{[29]}$ The pole density was calculated by the expression:

$$
P_{i}=\frac{\left(I_{i} / I_{0 i}\right)}{\frac{1}{N} \sum_{i=1}^{N}\left(I_{i} / I_{0 i}\right)}
$$

where $I_{i}$ and $I_{O i}$ are the integrated intensities of $i$-th diffraction peak for the sample film and etalon, respectively, and $N$ is the number of lines in the diffraction pattern.

Then, the dependences $P_{i}-(\mathrm{hkl})_{i}$ and $P_{i}-\varphi$ were built, where $\varphi$ is the angle between the texture axis and perpendicular to different crystal planes that correspond to reflections on diffraction patterns, and (hkl) is Miller indices. This angle was calculated for a hexagonal lattice using expressions given in ${ }^{[29]}$. Texture axis has those indices that correspond to the maximum value of $P_{i}$. In this case, the orientation factor for the corresponding sample can be found from the expressionf $=\sqrt{\frac{1}{N} \sum_{i=1}^{N}\left(P_{i}-1\right)^{2}}$.

The calculation of the hexagonal phase constants $a$ and $c$ was carried out using the formulas:

$$
\begin{aligned}
& a=\frac{\lambda}{2 \sin \theta} \sqrt{\frac{4}{3}\left(h^{2}+h k+k^{2}\right)+\left(\frac{a}{c}\right)^{2} l^{2}} \\
& c=\frac{\lambda}{2 \sin \theta} \sqrt{\frac{4}{3}\left(\frac{c}{a}\right)^{2}\left(h^{2}+h k+k^{2}\right)+l^{2}}
\end{aligned}
$$

where $c / a=1.602$.

The lattice parameters were determined by the extrapolated Nelson-Riley method. ${ }^{[30,31]}$ For this matter, the graphs were built in the coordinates $a(c)-\frac{1}{2} \cos ^{2} \theta\left(\frac{1}{\sin \theta}+\frac{1}{\theta}\right)$. The linear approximation of obtained values was carried out using the least-squares method with the help of OriginPro software package (OriginLab Corporation, Northampton, MA, USA). The graphic method of consecutive approximations that included iteration procedure was used to increase the precision of the lattice parameters evaluation. It should be noted that the $c / a$ ratio was taken 1.602 for the first iteration. The values of items in Eqn 2, 3 were thus calculated for every diffraction line, and those with minimal contribution of the item with unknown value $(a / c$ and $c / a$ ) were selected to further find the lattice parameters $a$ and $c$. Then, from the graphs $a(c)-1$ / $2 \cos ^{2} \theta(1 / \sin (\theta)+1 / \theta)$, the lattice parameters $a$ and $c$ and their ratio were determined. The corresponding values of $c / a(a / c)$ were used for a new iteration of Eqn (2) and (3). The iteration procedure was repeated a few times (up to five) until the obtained values $a$ and $c$ and $c / a$ stopped changing. The described method allows finding lattice parameters of the material with $0.001 \%$ accuracy.

The average coherent scattering domain size (CSD) $L$ was calculated using Sherrer's relation: ${ }^{[29]}$

$$
L=\frac{k \cdot \lambda}{\beta \cdot \cos \theta}
$$

where $k$ is a coefficient that depends on the grain size $(k=0.9)$, and $\beta$ is the physical broadening of corresponding $x$-ray lines. The 

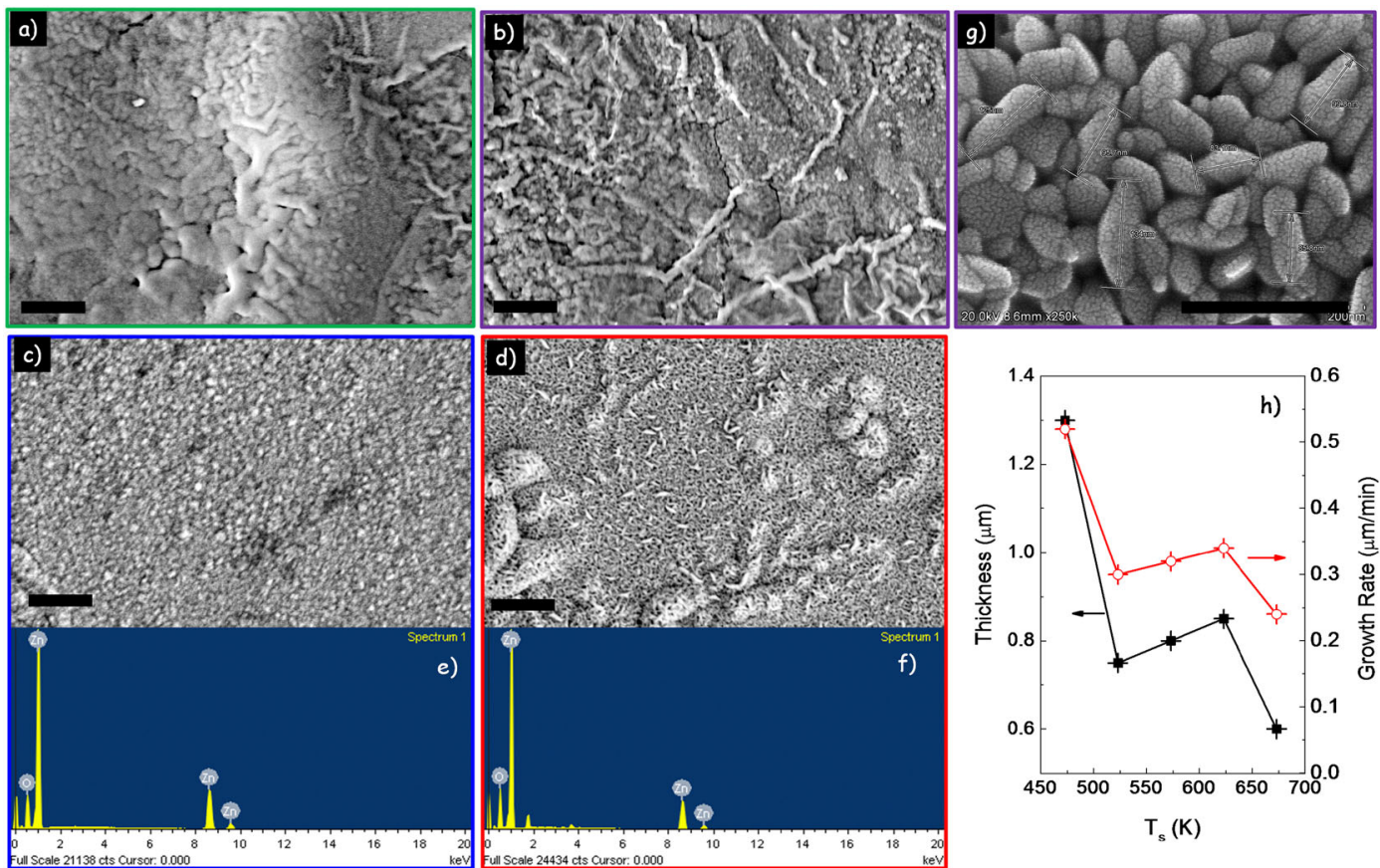

Figure 2. (a)-(d) SEM images of ZnO films deposited onto glass substrates at temperatures $T_{s}, \mathrm{~K}: 473$ (a), 573 (b), 623 (c), and 673 (d). The scale bars correspond to $2 \mu \mathrm{m}$; (e)-(f) EDX spectra from films obtained at $623 \mathrm{~K}$ (e) and $673 \mathrm{~K}(\mathrm{f}) ;(\mathrm{g})$ higher magnification SEM images of sample obtained at $573 \mathrm{~K}$. The scale bar corresponds to $200 \mathrm{~nm}$; and (h) thickness and growth rate as a function of $T_{s}$.

determination of the size of CSDs in films by the Scherer's formula using physical broadening of $x$-ray lines is used in many works. ${ }^{[33-35]}$ Indeed, the physical broadening of $x$-ray lines in addition to the small size of CSD is also due to the presence of microstrains, that is, crystallographic disorder and defects in the material. However, as we have shown in several papers, ${ }^{[36-38]}$ contributions of microstrains (associated with the presence of dislocations in the (SD) to the broadening of $x$-ray lines are substantially less than the dispersion of CSD (associated with the presence of dislocations at the boundaries of (SD). This particularly is applicable to the nanocrystalline films that were considered in this work. It has allowed us to neglect other causes of broadening of x-ray lines and to use the Scherer's formula.

Optical properties of the films were studied with a spectrophotometer PerkinElmer Lambda 950 (PerkinElmer, Waltham, MA, USA) in the range of wavelength $\lambda=(350-800) \mathrm{nm}$ at room temperature. The absorption coefficient $\alpha(\lambda)$ was calculated from the transmission spectra of $\mathrm{ZnO}$ film $T(\lambda) .^{[39]}$ The $\mathrm{ZnO}$ optical band gap $E_{g}$ was found from the absorption spectra using the following correlation:

$$
\alpha h v=A\left(h v-E_{g}\right)^{1 / 2}
$$

\begin{tabular}{|c|c|c|c|c|}
\hline$T_{s}, \mathrm{~K}$ & $C_{z n}$, at $\%$ & $C_{O}$, at $\%$ & $\gamma$ & $E_{g}, \mathrm{eV}$ \\
\hline 473 & 41.8 & 58.2 & 1.39 & 3.30 \\
\hline 523 & 42.3 & 57.7 & 1.36 & 3.27 \\
\hline 573 & 42.6 & 57.4 & 1.35 & 3.24 \\
\hline 623 & 44.3 & 55.7 & 1.26 & 3.18 \\
\hline \multirow{2}{*}{$\begin{array}{l}673 \\
{[46]}\end{array}$} & 44.0 & 56.0 & 1.27 & 3.21 \\
\hline & & - & & 3.37 \\
\hline
\end{tabular}

where $\alpha$ is the absorption coefficient; $h v$ is the photon energy, and $A$ is a constant that depends on the effective mass of the charge carriers in the material. Extrapolation of the linear part of graph $(\alpha h v)^{2}-h v$ on energy axis allowed determining the semiconductor band gap $E_{g}$.

\section{Results and discussion}

Figure 2 shows representative SEM micrographs of ZnO films deposited at different substrate temperatures. Solid films with good adhesion to substrate and no cracks were formed at temperatures as low as $473 \mathrm{~K}$ and above.

Figure $2 \mathrm{~h}$ shows the dependence of the substrate temperature on the thickness and growth rate of $\mathrm{ZnO}$ films. While the film

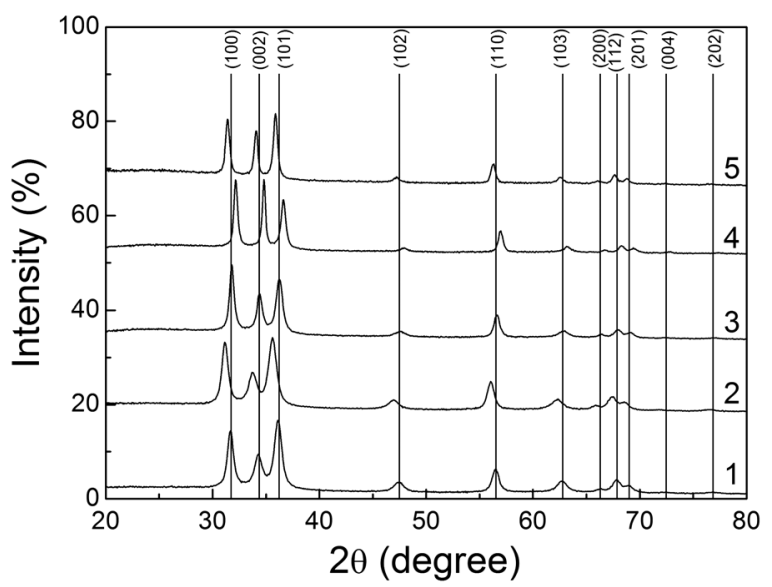

Figure 3. Diffraction patterns of $\mathrm{ZnO}$ films obtained at different substrate temperatures, $T_{s}, \mathrm{~K}: 473$ (1), 523 (2), 573 (3), 623 (4), and 673 (5). Vertical lines correspond to Joint Committee on Powder Diffraction Standards data (card Number 01-089-1397). 

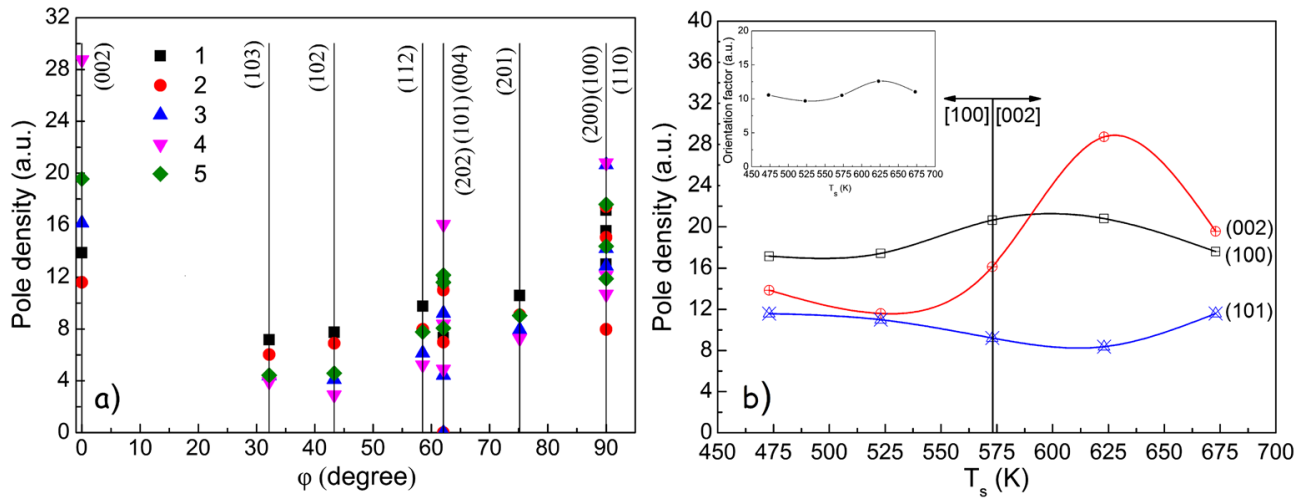

Figure 4. Dependence of the pole density Pi on the angle $\varphi$ between the texture axis and normal to the reflecting plane (a) and substrate temperatures (b), $T_{s}, \mathrm{~K}: 473(1), 523(2), 573(3), 623(4)$, and 673 (5). The inset (b) shows the dependence of $t\left(T_{s}\right)$.

thickness could be generally controlled by the number of pulses, it was found that the substrate temperature also had a strong influence on the growth rate and thus on the thickness of the films obtained at a set growth time. Setting the number of pulses to 30 cycles, thus a total growth time of $150 \mathrm{~s}$, the film thicknesses obtained were in the range from 0.6 to $1.3 \mu \mathrm{m}$, depending on the growth temperature.

High resolution SEM analysis of the samples allowed to determine the grain sizes of the $\mathrm{ZnO}$ films to be in the range 20-300 nm (Fig. 2g). The grain size increased with the substrate temperature. Figures $2 \mathrm{e}$ and $2 \mathrm{f}$ display representative EDX spectra from the synthesized $\mathrm{ZnO}$ films. The chemical composition obtained for the $\mathrm{ZnO}$ layers deposited at different substrate temperatures is summarized in Table 1. All the films had some surplus of oxygen, which concentration decreased with increasing substrate temperature from $\gamma=1.39$ at $T_{s}=473 \mathrm{~K}$ to $\gamma=1.26$ at $T_{s}=623 \mathrm{~K}$. This oxygen excess is related with the oxygen species adsorbed at the surface of the $\mathrm{ZnO}$ grains. No other impurities were identified in the samples when using zinc acetate as the $Z n$ precursor. Similar results were obtained by I. Stambolova et al. and R. Mariappan et al. ${ }^{[40,41]}$

Figure 3 shows the diffraction patterns of $\mathrm{ZnO}$ films obtained at different substrate temperatures. As a reference, the hexagonal ZnO phase, Joint Committee on Powder Diffraction Standards card number 01-089-1397, is also plotted. From these diffraction patterns, the hexagonal phase of the $\mathrm{ZnO}$ films formed was evidenced, and no secondary phase could be identified.

As it is seen from Fig. 3, the peak at $2 \theta \sim 36^{\circ}$ is dominant on the diffraction patterns of the films obtained at low temperature. This peak corresponds to the reflection from the (101) plane of the $\mathrm{ZnO}$ hexagonal phase. In the films obtained at $T_{s}>573 \mathrm{~K}$, the peaks

Table 2. The calculation results of the lattice parameters by NelsonRiley method of $\mathrm{ZnO}$ films deposited at different substrate temperatures

\begin{tabular}{|c|c|c|c|c|c|c|}
\hline \multirow[t]{2}{*}{$T_{s}, \mathrm{~K}$} & \multicolumn{3}{|c|}{1 iteration } & \multicolumn{3}{|c|}{5 iteration } \\
\hline & $a, \mathrm{~nm}$ & $c, \mathrm{~nm}$ & $c / a$ & $a, \mathrm{~nm}$ & $c, \mathrm{~nm}$ & $c / a$ \\
\hline 473 & 0.32524 & 0.52002 & 1.599 & 0.32543 & 0.51957 & 1.597 \\
\hline 523 & 0.32496 & 0.51880 & 1.597 & 0.32554 & 0.51507 & 1.582 \\
\hline 573 & 0.32461 & 0.51906 & 1.599 & 0.32494 & 0.51693 & 1.591 \\
\hline 623 & 0.32483 & 0.52095 & 1.604 & 0.32477 & 0.52111 & 1.605 \\
\hline \multirow{2}{*}{$\begin{array}{l}673 \\
{[28]}\end{array}$} & 0.32524 & 0.52002 & 1.599 & 0.32543 & 0.51957 & 1.597 \\
\hline & \multicolumn{6}{|c|}{$a=0.3253 \mathrm{~nm}, c=0.5213 \mathrm{~nm}$, and $c / a=1.602$} \\
\hline
\end{tabular}

at $2 \theta \sim 31.80^{\circ}$ and $34.80^{\circ}$ dominate. These correspond to the reflections from crystallographic planes (100) and (002), respectively.

The position of the peaks shifts towards lower angles when increasing the substrate temperature from $473 \mathrm{~K}$ to $523 \mathrm{~K}$ and then towards larger angles when increasing the substrate temperature up to $623 \mathrm{~K}$. The peaks shift again towards lower angles when

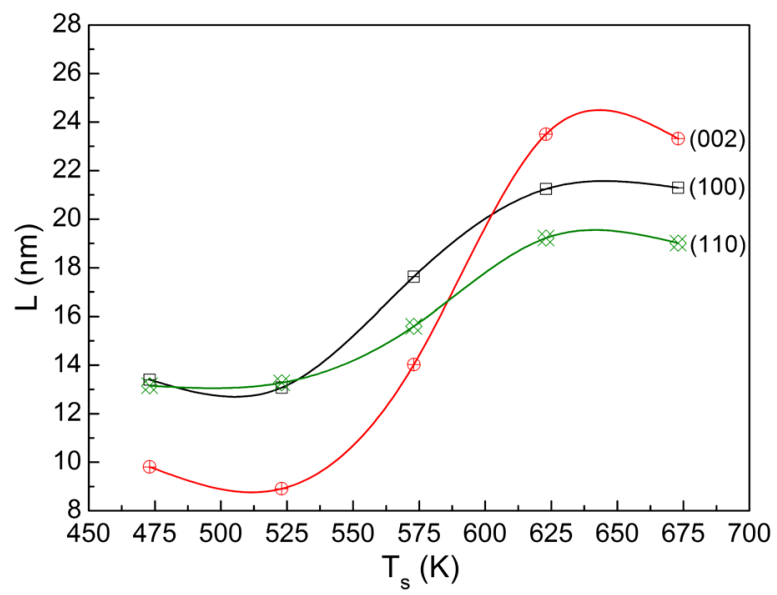

Figure 5. Dependence of coherent scattering domain sizes $(L)$ of $\mathrm{ZnO}$ films on the substrate temperatures.

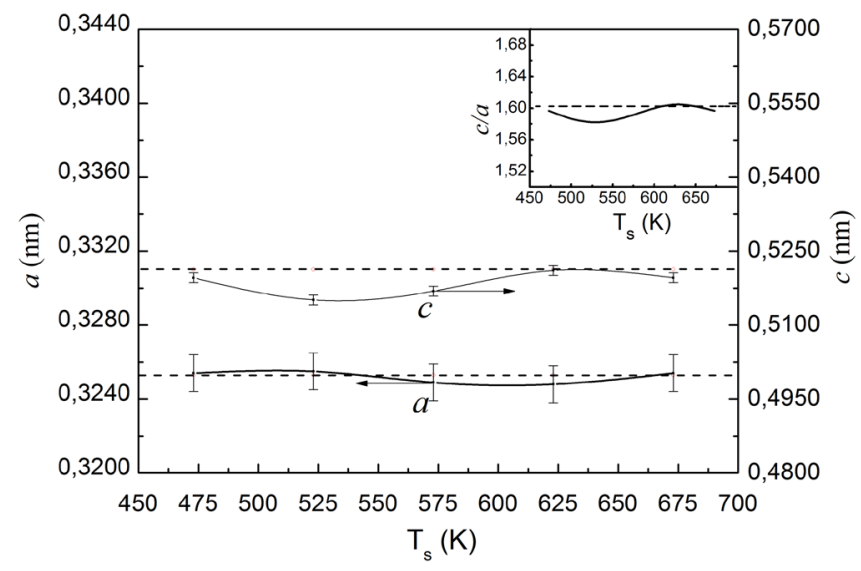

Figure 6. Dependence of the lattice parameter $a$ and ratio $c / a$ on the substrate temperatures (fifth iretation). 

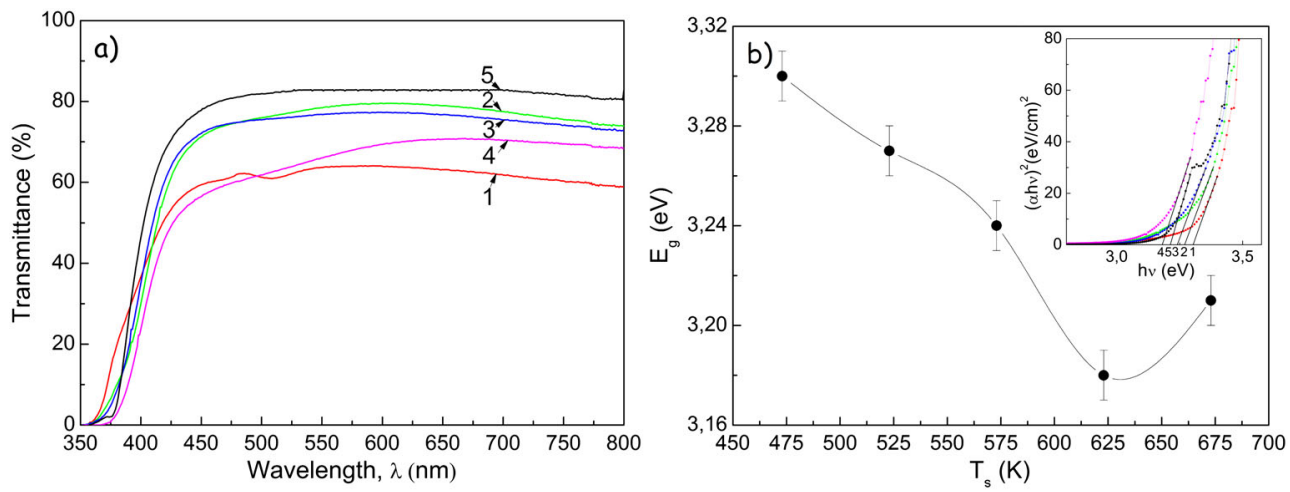

Figure 7. Transmission spectra of ZnO films (a) and the dependence of the band gap (b) on the substrate temperatures, $T_{s}, \mathrm{~K}: 473$ (1), 523 (2), 573 (3), 623 (4), and $673(5)$.

increasing the deposition temperature from $623 \mathrm{~K}$ to $673 \mathrm{~K}$. This complex pattern can be explained by a combination of the compositional evolution of the material and presence of residual stresses in the films as previously observed in. ${ }^{[42,43]}$ These residual stresses could be caused by both the lattice mismatch and thermal expansion coefficient difference between film and substrate. ${ }^{[44]}$ The peaks' shift towards lower and higher angles means that the lattice constant $c$ decreases and increases with increasing the substrate temperature, respectively, which are considered to be related to the residual stress in the $\mathrm{ZnO}$ films.

Calculations of the pole density $P_{i}$ (Fig. 4a) allowed to determine an axial texture growth in the (100) direction in $\mathrm{ZnO}$ layers obtained at $T_{s}<573 \mathrm{~K}$. This texture changed into a texture in the (002) direction at $T_{s}>573 \mathrm{~K}$ (Fig. 4b). In the inset of Fig. $4 \mathrm{~b}$, the dependence of the orientation factor $f\left(T_{s}\right)$ of the films on the substrate temperature is given. The orientation factor increased with $T_{s}$, which confirmed the improvement of the film texture quality. The decreasing $f$ in the layers obtained at $623 \mathrm{~K}$ was connected with the change of texture direction from (100) to (002).

The results obtained from calculating the average CSD sizes of $\mathrm{ZnO}$ films in directions perpendicular to the crystallographic planes (100), (110), and (002) are shown in Fig. 5 as a function of the substrate temperature. The average CSD size for (100) increased from $L \sim 10$ to $L \sim 20 \mathrm{~nm}$ in the three directions. This increase of the CSD is associated both to an increase of the crystal domain size and to an improved crystalline quality of the films. When the substrate temperature increases, the size of CSD in the films also increases, and accordingly, it reduces the concentration of dislocations. Thus, the films deposited at higher temperatures have larger defect-free crystalline regions, which reduced the structural imperfections of the material.

The lattice parameter of the material is a property extremely sensitive to changes in its stoichiometry, impurities, oxidation, and others, which is why the precision measurement of these values makes it possible to study the relevant processes. With the help of the iteration procedure described previously, we carried out $x$-ray diffraction measurements of the lattice parameters $a, c$, and the ratio $c / a$ of $\mathrm{ZnO}$ films. The obtained values of the crystal lattice parameters of the material after the first and fifth iteration are shown in Table 2.

Figure 6 shows the dependences of the lattice parameters $a$ and $c$ and their ratio $c / a$ on the substrate temperature. The horizontal lines correspond to the reference values found in the literature for stoichiometric samples.
As shown in Fig. 6, already at $625 \mathrm{~K}$, the calculated values $a$ and $c$ approached the reference values, which can be associated with a good stoichiometry and crystallinity of the films. EDX also confirmed that the composition of high-temperature condensates approached the stoichiometric one.

As it is shown in Fig. 7a, the transmittance in the layers was $T=60-80 \%$ in the whole wavelength range above $450 \mathrm{~nm}$. It is important to note that the films with higher thickness showed reduced transmittance coefficients. Films obtained at $T_{s}=673 \mathrm{~K}$ had the highest transmittance values. The inset in Fig. 7b shows $(\alpha h v)^{2}$ against $h v$ for films that we used to determine the band gap of the material. The results are presented in Table 1.

The dependence of the obtained values of the band gap $E_{g}$ on the substrate temperature $T_{s}$ is shown in Fig. 7b. As shown, $E_{g}$ of the material decreased with increased $T_{s}$. This may be associated to the observed slight change of composition and particularly to a loss of hydroxyl groups from the $\mathrm{ZnO}$ film when increasing the substrate temperature. A similar trend was observed by Zahedi et al. ${ }^{[45]}$

The $\mathrm{ZnO}$ films deposited at the highest substrate temperatures tested here, $623-673 \mathrm{~K}$, showed thus the best characteristics to be used as window layers of thin film solar cells. Such films show a single hexagonal phase structure with relatively large grain size, high crystalline quality, and transparency.

\section{Conclusions}

Crystalline $\mathrm{ZnO}$ films were obtained by pulsed spray pyrolysis on glass substrates. The influence of substrate temperature on the grain size, phase composition, texture quality, CSD size, crystal lattice parameters, chemical composition, transmission coefficient, and the band gap of the material were studied. EDX analysis revealed the $\mathrm{ZnO}$ films had some surplus of oxygen. It was shown that the films with thicknesses ranging from $0.6 \mu \mathrm{m}$ to $1.3 \mu \mathrm{m}$ had a crystalline structure with grain size in the range from 20 to $300 \mathrm{~nm}$, increasing with the substrate temperature. Films showed a single $\mathrm{ZnO}$ hexagonal phase. An axial texture in the [100] direction was identified in the samples grown at $T_{s}<573 \mathrm{~K}$ and in the [002] direction at $T_{s}>573 \mathrm{~K}$. It was established that with the change of substrate temperature, the CSD size of the films increased in the range from $10 \mathrm{~nm}$ up to $25 \mathrm{~nm}$. Moreover, the experimental values of the lattice parameters of the material were calculated, $a=0.32477-0.32554 \mathrm{~nm}, c=0.51507-0.52111 \mathrm{~nm}$, and 
$c / a=1.582-1.605$ and were correlated well with the reference data. The band gap of the films was found in the range $E_{g}=3.18-3.30 \mathrm{eV}$.

\section{Acknowledgements}

This research was supported by the Ministry of Education and Science of Ukraine (Grant Numbers 0113U000131, 0112U000772, and the individual grant for D.O.).

\section{References}

[1] U. A. Ozgur, Y. I. Alivov, A. Teke, et al., J. Appl. Phys. 2005, 98, 041301.

[2] V. V. Kireev, L. N. Dem'yanets, L. E. Li, et al., Inorg. Mater. 2010, 46, 154.

[3] T. V. Semikina, L. N. Shmyreva, Electron. Connect. Thematic issue "Electron. Nanotechnol." 2010, 3, 20.

[4] D. C. Look, Mater. Sci. Eng. B 2001, 80, 383.

[5] P. S. Shewale, G. L. Agawane, S. W. Shin, et al., Sensor. Actuat. B-Chem 2013, 177, 695.

[6] C. S. Prajapati, P. P. Sahay, J. Nano-Electron. Phys. 2011, 3, 714.

[7] S. Gledhill, A. Grimm, N. Allsop, et al., Thin Solid Films 2009, 517, 2309.

[8] A. Crossay, S. Buecheler, L. Kranz, et al., Sol. Energ. Mat. Sol. C. 2012, 101, 283.

[9] C. M. Mahajan, M. G. Takwale, J. Alloy. Compd. 2014, 584, 128.

[10] C. Levy-Clement, R. Tena-Zaera, M. A. Ryan, et al., Adv. Mater. 2005, 17, 1512.

[11] Y.-P. Liao, J.-H. Zhang, S.-X. Li, et al., Phys. Status Solidi A 2010, 207, 1850.

[12] S. Fay, U. Kroll, C. Bucher, et al., Sol. Energ. Mat. Sol. C. 2005, 86, 385.

[13] S. A. Aly, N. Z. El-Sayed, M. A. Kaid, Vacuum 2001, 61, 1.

[14] A. S. Opanasyuk, T. O. Berestok, P. M. Fochuk, et al., SPIE Proc. Ser. 2013, $8823,88230 \mathrm{Q}$.

[15] A. Khoury, R. al Asmar, M. Abdallah, et al., Phys. Status Solidi A 2010, 207, 1900.

[16] M. Amlouk, F. Touhari, S. Belgacem, et al., Phys. Status Solidi A 1997, 163, 73.

[17] J. Fan, Y. Hao, A. Cabot, et al., ACS Appl. Mater. Inter. 2013, 5, 1902.

[18] J. Fan, Y. Hao, C. Munuera, et al., J. Phys. Chem. C 2013, 117, 16349.

[19] J. D. Fan, C. Fabrega, R. Zamani, et al., J. Alloys Compd. 2013, 555, 213.

[20] J. Fan, R. Zamani, C. Fábrega, et al., J. Phys. D Appl. Phys. 2012, 45, 415301.
[21] J. Fan, F. Güell, C. Fábrega, et al., J. Phys. Chem. C 2012, 116, 19496.

[22] J. Fan, F. Guell, C. Fábrega, et al., Appl. Phys. Lett. 2011, 99, 262102.

[23] J. D. Fan, A. Shavel, R. Zamani, et al., Acta Mater. 2011, 59, 6790.

[24] A. Ashour, M. A. Kaid, N. Z. El-Sayed, et al., Appl. Surf. Sci. 2006, 252, 7844.

[25] R. Ayouchi, D. Leinen, F. Martin, et al., Thin Solid Films 2003, 426, 68

[26] M. Ortel, T. Balster, V. Wagner, J. Appl. Phys. 2013, 114, 234502.

[27] O. A. Dobrozhan, A. S. Opanasyuk, S. B. Bolshanina, Odes'kyi Politechnichnyi Universytet. Pratsi 2013, 4, 173.

[28] Selected power diffraction data for education and training (search manual and data cards), International Center for Diffraction Data, Pennsylvania, USA: published by the International Centre for Diffraction Data, 1988.

[29] B. E. Warren, X-ray Diffraction, Dover, New York, 1990.

[30] D. Kurbatov, H. Hlyap, A. Opanasyuk, Phys. Status Solidi A 2009, 206, 1549.

[31] D. Kurbatov, V. Kosyak, M. Kolesnyk, et al., Integr. Ferroelectr. 2008, 10, 32.

[32] D. Kurbatov, A. Opanasyuk, S. M. Duvanov, et al., Solid State Sci. 2011, $13,1068$.

[33] R. Sridhar, C. Manoharan, S. Ramalingam, et al., Spectrochim. Acta A 2014, 120, 297.

[34] F. Zahedi, R. S. Dariani, S. M. Rozati, Mat. Sci. Semicon. Proc. 2013, 16, 245.

[35] S. D. Shinde, G. E. Patil, D. D. Kajale, J. Alloy. Compd. 2012, 528, 109.

[36] A. S. Opanasyuk, D. I. Kurbatov, V. V. Kosyak, Crystallogr. Rep. 2012, 57, 927.

[37] V. Kosyak, A. Opanasyuk, P. M. Bukivskij, J. Cryst. Growth. 2010, 312, 1726.

[38] C. J. Panchal, A. S. Opanasyuk, V. V. Kosyak, J. Nano- Electron. Phys. 2011, 3, 274.

[39] R. Mariappan, V. Ponnuswamy, A. Chandra Bose, et al., Superlattice. Microst. 2014, 65, 184

[40] I. Stambolova, V. Blaskov, M. Shipochka, et al., Mater. Sci. Eng. B 2012, 177, 1029.

[41] R. Mariappan, V. Ponnuswamy, M. Ragavendar, et al., Mat. Sci. Semicon. Proc. 2013, 16, 1328.

[42] U. Alver, A. Kudret, S. Tekerek, J. Phys. Chem. Solids 2011, 72, 701.

[43] H. L. Ma, Z. W. Liu, D. C. Zenga, et al., Appl. Surf. Sci. 2013, 283, 1006.

[44] J.-W. Jeon, M. Kim, L.-W. Jang, J. L. Hoffman, et al., Electron. Mater. Lett. 2012, 8, 27.

[45] F. Zahedi, R. S. Dariani, S. M. Rozati, Bull. Mater. Sci. 2014, 37, 433.

[46] R. Ayouchi, F. Martin, D. Leinen, et al., J. Cryst. Growth 2003, 247, 497. 